\title{
Is there a place for anti-nucleosome antibody assessment in scleroderma?
}

\author{
Alexandra Burlui ${ }^{1,2}$, Anca Cardoneanu ${ }^{1,2}$, LuanaAndreea Macovei ${ }^{1,2}$, \\ LidiaArhire ${ }^{1}$, Mariana Graur ${ }^{1}$, Elena Rezus ${ }^{1,2}$ \\ ${ }^{1}$ Faculty of Medicine, Department of Medical Sciences II, \\ "Grigore T. Popa" University of Medicine and Pharmacy, Iasi, Romania \\ ${ }_{2}^{2}{ }^{\text {st }}$ Rheumatology Clinic, Clinical Rehabilitation Hospital, Iasi, Romania
}

\begin{abstract}
Introduction. The hallmarks of systemic sclerosis (SSc) include microangiopathy, autonomic dysfunction, as well as immune disturbance and the widespread fibrosis of the skin and visceral organs. While the significance of SSc-specific autoantibodies such as anti-centromere and anti-topoisomerase I has long been demonstrated, the clinical relevance of non-specific autoantibodies remains a matter of debate. Our primary objective was to assess the relationships between non-SSc-specific antibody titers and the clinical characteristics of scleroderma patients. Secondary objectives included a comparison between SSc, SLE and healthy controls (HC) with respect to autoantibody values, as well as the analysis of the immune disturbance in elderly individuals in the 3 groups. Material and method. We conducted a cross-sectional study in which we recruited 67 adult patients with SSc, 67 age and gender-matched individuals with SLE and healthy controls (HC). Biological samples (venous blood) were collected in order to determine the levels of anti-SSA/Ro, anti-SSB/La, anti-U1RNP and anti-nucleosome antibodies (ELISA). We recorded the presence of digital ulcers (DUs), ILD (thoracic X-rays), and PAH (Doppler echocardiography) in the scleroderma cohort.

Results. The frequency of anti-nucleosome antibody positivity in the scleroderma group exceeded our expectations, resembling that of lupus patients. Moreover, our findings indicate an association between serum anti-nucleosome antibody titers and SSc-related cardiopulmonary involvement. Anti-U1RNP antibodies were linked to PAH. We did not identify a notable relationship between the 4 autoantibodies studied and DUs. However, the latter were significantly more frequent in male patients. Although elderly individuals with scleroderma did not demonstrate a significantly decreased autoantibody production, lupus patients over 60 years of age exhibited a decline in anti-nucleosome antibody titers.

Discussions. Earlier research reported an association between anti-nucleosome and anti-U1RNP antibodies with SSc-related cardiopulmonary impairment. Moreover, male gender is currently regarded as an important risk factor for the development of scleroderma DUs.

Conclusions. Recent research provides new insights on the pathogenic processes of autoimmune rheumatic diseases, in an attempt to identify potential risk factors for organ involvement. Our study confirms the link between anti-nucleosome antibodies and cardiopulmonary involvement in the SSc population. Moreover, the impact of immunosenescence on the dynamics of autoantibody production in connective tissue diseases remains in need of further investigation.
\end{abstract}

Keywords: systemic sclerosis, systemic lupus erythematosus, nucleosome, immunosenescence, pulmonary arterial hypertension

\section{INTRODUCTION}

Connective tissue diseases (CTD) constitute a cluster of autoimmune disorders frequently associated with severe multi-organ involvement. Admitting that their intricate pathogenic mechanisms are yet to be fully understood, immune processes remain of paramount importance. Future directions involve a possible molecular-based reclassification of systemic autoimmune rheumatic diseases $(1,2)$.
Systemic sclerosis ( $\mathrm{SSc}$ ) is a chronic autoimmune condition exhibiting an elevated risk of morbidity and mortality. The hallmarks of scleroderma include microangiopathy, autonomic dysfunction, as well as immune disturbance and the widespread fibrosis of the skin and internal organs (3). The positivity of SSc-specific autoantibodies often indicates the phenotype of the disease, along with potential organ involvement. Patients demonstrating high cir- 
culating values of anti-topoisomerase I and anti-RNA polymerase III antibodies most commonly present with diffuse cutaneous involvement, whereas increased anti-centromere antibodies are typical of the limited form of disease. Nonetheless, a wide spectrum of non-specific autoantibodies have been described in SSc, with uncertain effects on disease pathomechanisms $(4,5)$. Earlier research reported a relationship between anti-nucleosome autoantibodies and localized scleroderma (6).

It has been shown that autoantibody production in CTD is influenced by numerous factors including the process of aging. In this respect, the concept of immunosenescence describes a decreased capacity for antibody production by plasma cells in elderly populations. However, whether or not these changes are clinically relevant in CTD currently remains a subject of debate $(7,8)$.

Regarded as the prototype of autoimmune disease, a great number of studies compared SLE antibody levels to the ones detected in other connective tissue disorders, reporting discrepant findings $(9,10)$. The complex immune disturbance associated with SSc pathogenesis has lead to extensive research in the field (11). Our primary aim was to assess the relationships between non-SSc-specific antibody titers and certain clinical characteristics of scleroderma patients. Secondary objectives included a comparison between SSc, SLE and healthy controls (HC) with respect to autoantibody values, as well as the analysis of the immune disturbance in elderly individuals in the 3 groups.

\section{MATERIAL AND METHODS}

We conducted a cross-sectional study in which we recruited 67 adult patients with SSc hospitalized in our center between september 2014 and september 2017, 67 age and gender-matched individuals with SLE and healthy controls (HC). For the SSc group, the inclusion criteria were the following: age $\geq 18$ years and confirmed diagnosis of systemic sclerosis. The exclusion criteria were age $<18$ years, overlap syndromes and scleroderma sine scleroder$m a$. The corresponding SLE (definite diagnosis) and $\mathrm{HC}$ groups were matched to the scleroderma cohort with respect to age and gender. We excluded SLE patients with overlap syndromes.

Biological samples (venous blood) were collected in order to determine the levels of anti-SSA/Ro, anti-SSB/La, anti-U1RNP and anti-nucleosome anti- bodies. All autoantibodies were assessed by enzyme-linked immunoassay (ELISA). We recorded the presence of DUs, ILD (thoracic X-rays), and PAH (Doppler echocardiography). The analysis of the data was performed using Microsoft Office Excel and IBM SPSS Statistics v20 for Windows. We used either parametric or non-parametric tests depending on variable distribution. In the contingency tables, we applied the chi ${ }^{2}$ test and Fisher's exact test (where chi ${ }^{2}$ did not demonstrate statistical consistency).

\section{RESULTS}

Our cohort consisted of 67 patients with scleroderma, of which 32 were classified as dcSSc (47.8\%) while 35 persons presented with $1 \mathrm{cSSc}(52.2 \%)$. The group was composed of 56 women $(83.6 \%)$ and 11 men (16.4\%). We identified the presence of ILD in 24 patients (35.8\%), PAH in 5 patients $(7.5 \%)$ and DUs in 29 patients $(43.3 \%)$.

Male participants were more likely to present with digital trophic lesions than females $(72.7 \%$ vesus $37.5 \%, p=0.031)$, while elderly patients $(\geq 60$ years of age, 19 patients) demonstrated an increased risk of ILD by $113.8 \%(\mathrm{RR}=2.138,95 \% \mathrm{CI}: 1,171$ $3,903)$ and $910,5 \%$ for PAH $(\mathrm{RR}=10,105,95 \% \mathrm{CI}$ : 1,206-84,690).

The values of the four autoantibodies tested are illustrated in Table 1. With regard to disease phenotype, we found statistically significant discrepancies between the dcSSc and $\mathrm{lcSSc}$ patients in relation to the serum levels of anti-SSA antibodies (student t-test, $p=0.014$ ). Furthermore, patients with dcSSc were more likely to exhibit anti-SSA positivity $(34.4 \%$ versus $5.7 \%, \mathrm{p}=0.003)$.

TABLE 1. Autoantibody values in patients with scleroderma

\begin{tabular}{|l|c|c|c|c|}
\hline $\begin{array}{l}\text { Autoantibodies } \\
\text { (U/ml) }\end{array}$ & Range & Mean & SD & $\begin{array}{c}\text { P } \\
\text { (student } \\
\text { t-test) }\end{array}$ \\
\hline $\begin{array}{l}\text { Anti-SSA } \\
\text { dcSSc }\end{array}$ & $0.00-932.00$ & 112.42 & 227.83 & \\
IcSSc & $0.00-905.00$ & 182.84 & 270.46 & $\mathbf{0 . 0 1 4}$ \\
\hline Anti-SSB & $0.00-932.00$ & 48.03 & 158.53 & \\
dcSSc & $0.00-938.00$ & 59.34 & 182.94 & \\
IcSSc & $0.00-938.00$ & 95.47 & 238.77 & 0.125 \\
\hline Anti- & $0.00-614.00$ & 26.69 & 103.22 & \\
nucleosome & $0.00-367.00$ & 109.18 & 96.44 & \\
dcSSc & $0.00-367.00$ & 110.69 & 102.69 & 0.904 \\
IcSSc & $0.00-349.00$ & 107.80 & 91.83 & \\
\hline Anti-RNP/Sm & $0.00-126.00$ & 21.40 & 36.37 & \\
dcSSc & $0.00-126.00$ & 23.72 & 24.14 & 0.493 \\
IcSSc & $0.00-114.00$ & 19.28 & 28.44 & \\
\hline
\end{tabular}

*SD = standard deviation 
Anti-SSB, anti-U1RNP, and anti-nucleosome antibodies had slightly higher titers in dcSSc patients compared to the opposing subgroup. However, these findings did not demonstrate statistical significance.

We found higher circulating anti-nucleosome antibodies in persons with PAH $(186.60 \pm 55.66 \mathrm{U} / \mathrm{ml}$ versus $102.92 \pm 96.57 \mathrm{U} / \mathrm{ml}$; Mann-Whitney, $\mathrm{p}=0.030$ ) (Figure 1). Moreover, patients wih positive anti-nucleosome antibodies were likely to have PAH (Fisher's exact test, $\mathrm{p}=0.018$ ). The same was true for anti-U1RNP antibody positivity and PAH (Fisher's exact test, $\mathrm{p}=0.013$ ), as well as ILD (Fisher's exact test, $\mathrm{p}=0.042$ ). Patients with DUs also demonstrated elevated anti-nucleosome antibody titers (132.52 \pm $111.81 \mathrm{U} / \mathrm{ml}$ versus $91.37 \pm 79.84 \mathrm{U} / \mathrm{ml})$, but results were not statistically significant $(\mathrm{p}=0.099)$.

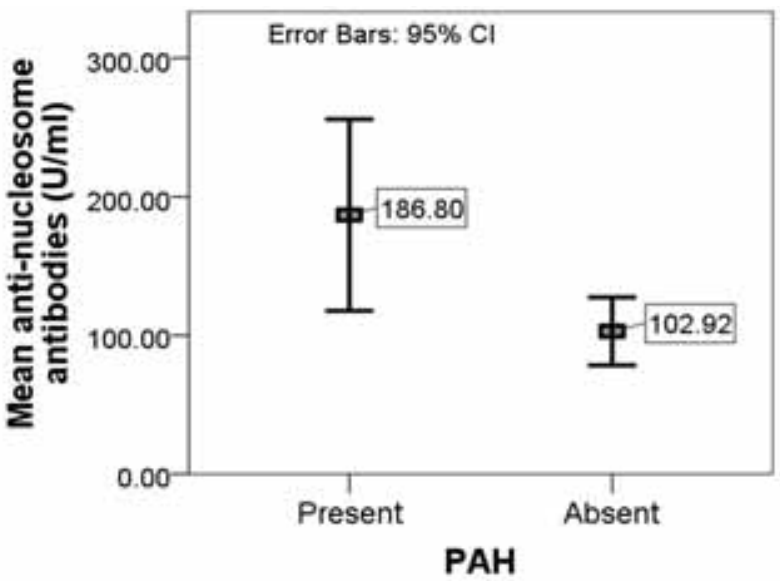

FIGURE 1. Anti nucleosome antibodies in scleroderma patients with/without $P A H$

We did not identify a notable relationship between age and specific autoantibody titers in the SSc group, with similar values in patients over and under 60 years of age (Table 2 ).

TABLE 2. Autoantibody titers in scleroderma patients over and under 60 years of age

\begin{tabular}{|c|c|c|c|c|c|}
\hline \multicolumn{2}{|c|}{$\begin{array}{l}\text { Autoantibody titer } \\
(\mathrm{U} / \mathrm{ml})\end{array}$} & \multirow{2}{*}{\begin{tabular}{|l} 
Range \\
$0.00-932.00$ \\
\end{tabular}} & \multirow{2}{*}{\begin{tabular}{|l|} 
Mean \\
72.47 \\
\end{tabular}} & \multirow{2}{*}{\begin{tabular}{|l|} 
SD \\
211.88 \\
\end{tabular}} & \multirow{2}{*}{$\begin{array}{c}\mathrm{p} \\
\text { (student } \\
\text { t-test) }\end{array}$} \\
\hline 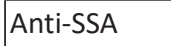 & $\geq 60$ years & & & & \\
\hline & $<60$ years & $0.00-905.00$ & 128.23 & 234.09 & \\
\hline \multirow[t]{2}{*}{ Anti-SSB } & $\geq 60$ years & $0.00-614.00$ & 48.79 & 144.43 & \multirow[t]{2}{*}{0.733} \\
\hline & $<60$ & 0.00 & 63.79 & 197.33 & \\
\hline \multirow{2}{*}{\begin{tabular}{|l|} 
Anti- \\
Nucleosome \\
\end{tabular}} & $\geq 60$ & 0. & 133.32 & 104.19 & \multirow[t]{2}{*}{0.770} \\
\hline & $<60$ years & $0.00-126.00$ & 99.63 & 92.59 & \\
\hline \multirow[t]{2}{*}{ Anti-U1RNP } & $\geq 60$ years & $0.00-114.00$ & 19.74 & 30.49 & \multirow[t]{2}{*}{0.228} \\
\hline & $\mid<60$ years & $0.00-367.00$ & 22.06 & 24.88 & \\
\hline
\end{tabular}

*SD = standard deviation

Compared to the SLE group, patients with scleroderma exhibited lower anti-SSA, U1RNP and an- ti-nucleosome antibodes, while anti-SSB titers did not differ significantly. Moreover, all antibody titers were higher in SSc compared to HC (Table 3).

TABLE 3. Autoantibody titers in patients with scleroderma compared to SLE and HC

\begin{tabular}{|c|c|c|c|c|c|}
\hline & $\begin{array}{l}\text { Mini- } \\
\text { mum }\end{array}$ & $\begin{array}{l}\text { Maxi- } \\
\text { mum }\end{array}$ & Mean & SD & $\begin{array}{c}\mathrm{p} \\
\text { (student } \\
\text { t-test) }\end{array}$ \\
\hline \multicolumn{6}{|c|}{ Age (years) } \\
\hline SSC & 33.00 & 80.00 & 51.51 & 11.54 & - \\
\hline SLE & 31.00 & 77.00 & 51.30 & 11.50 & 0.917 \\
\hline $\mathrm{HC}$ & 33.00 & 79.00 & 51.21 & 11.28 & 0.88 \\
\hline \multicolumn{6}{|c|}{ Autoantibodies (U/ml) } \\
\hline \multicolumn{6}{|c|}{ Anti-SSA } \\
\hline SSC & 0.00 & 932.00 & 112.42 & 227.83 & - \\
\hline SLE & 0.00 & 977.00 & 252.61 & 292.42 & 0.002 \\
\hline $\mathrm{HC}$ & 0.00 & 62.00 & 14.69 & 13.42 & 0.001 \\
\hline \multicolumn{6}{|c|}{ Anti-SSB } \\
\hline SSc & 0.00 & 938.00 & 59.34 & 182.94 & - \\
\hline SLE & 0.00 & 1061.00 & 74.64 & 183.06 & 0.634 \\
\hline $\mathrm{HC}$ & 0.00 & 83.00 & 8.19 & 14.92 & 0.025 \\
\hline \multicolumn{6}{|c|}{$\begin{array}{l}\text { Anti- } \\
\text { nucleosome }\end{array}$} \\
\hline SSc & 0.00 & 367.00 & 109.18 & 96.44 & - \\
\hline SLE & 0.00 & 1654.00 & 233.21 & 403.91 & 0.017 \\
\hline $\mathrm{HC}$ & 0.00 & 92.00 & 13.39 & 21.83 & $<0.001$ \\
\hline \multicolumn{6}{|c|}{ Anti-U1RNP } \\
\hline SSc & 0.00 & 126.00 & 21.4 & 36.37 & - \\
\hline SLE & 0.00 & 867.00 & 154.19 & 209.95 & $<0.001$ \\
\hline $\mathrm{HC}$ & 0.00 & 106.00 & 12.7 & 18.89 & 0.03 \\
\hline
\end{tabular}

Anti-U1 RNP antibodies, SSA and SSB were significantly more frequent in the SLE group compared to SSc (Figure 2). However, anti-nuclesosme antibodies demonstrated similar frequencies in the the two aforementioned groups ( $\mathrm{chi}^{2}$ test, $\mathrm{p}=0.547$ ).

Elderly patients in the entire cohort (patients with SSc, SLE and HC) did not demonstrate significantly lower autoantibody titers (student t-test, SSA: $\mathrm{p}=0.058$, SSB: $\mathrm{p}=0.258$, nucleosome: $\mathrm{p}=0.118$, U1RNP: $p=0.851)$. Anti-SSA antibodies approached statistical significance and were found to be higher in persons under 60 years of age $(146.28 \pm 249.96 \mathrm{U} /$ $\mathrm{ml}$ versus $76.77 \pm 182.54 \mathrm{U} / \mathrm{ml})$.

Moreover, elderly patients with lupus exhibited significantly lower anti-nucleosome antibody values compared to younger individuals belonging to the same group $(296.83 \pm 456.69 \mathrm{U} / \mathrm{ml}$ versus $72.47 \pm$ $125.64 \mathrm{U} / \mathrm{ml}, \mathrm{p}=0.039)$.

\section{DISCUSSION}

Our study aimed to analyze the relationship between non-specific antibodies and clinical presenta- 
SSc

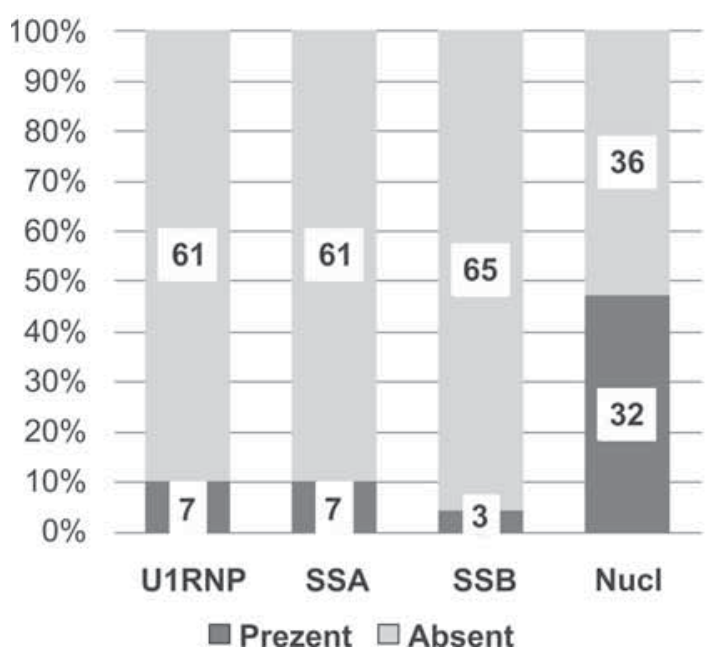

SLE

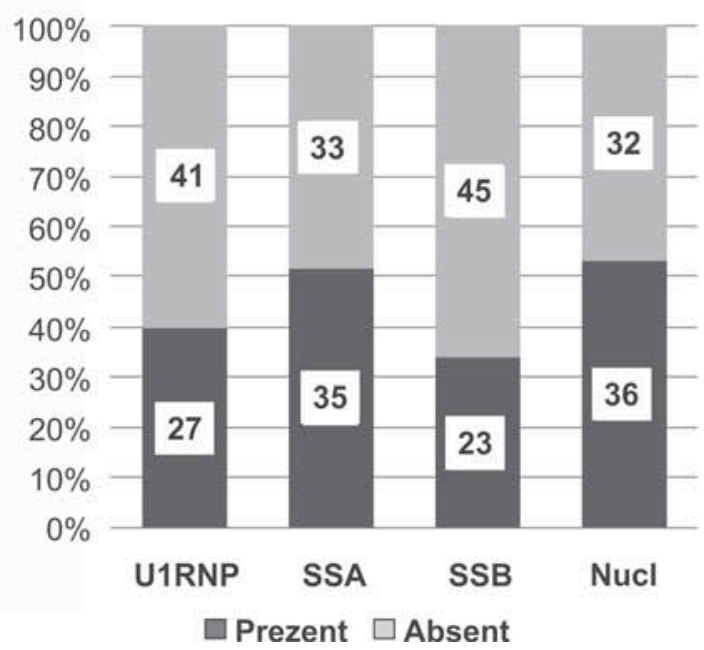

FIGURE 2. The frequency of autoantibody positivity in patients with SSc versus SLE

tion in SSc patients. Recently, the issue of finding new biomarkes in scleroderma has been gathering attention from the scientific community $(12,13)$. We recorded a higher prevalence of anti-U1RNP antibodies in our scleroderma group compared to other studies (9). The link between anti-U1RNP antibodies in CTD-related PAH (including SSc) has been described in previously published research $(14,15)$.

Furthermore, the enhanced expression of anti-nucleosome antibodies in SSc patients exceeded our expectations. Although the mean anti-nucleosome values were found to be lower in scleroderma patients compared to SLE in our study population, circa $46 \%$ of the SSc group were positive for these antibodies, similar to the SLE cohort. Whereas their importance in lupus has long been demonstrated, studies describing their high specificity for SLE, as well as their notable nephritogenic potential, the significance of anti-nucleosome antibody positivity in $\mathrm{SSc}$ is yet to be fully elucidated (16).

Admitting that our findings could be due to the potentially non-discriminative nature of anti-nucleosome antibodies and their consequent cross-reactivity with other structures, we also found an association with $\mathrm{PAH}$ in our cohort, suggesting a possible involvement in disease pathogensis (17).

Previous studies have described elevated anti-nucleosome antibody levels in SSc-related lung fibrosis (18-20). We did not identify a significant relationsip between anti-nucleosome antibodies and ILD in our SSc cohort. However, knowing that ILD may coexist with PAH in SSc and that high resolution computed tomography for ILD and right heart angiography for PAH demonstrate higher sensitivity in the detection of SSc-related cardiopulmonary involvement compared to the methods used in the present study, we recognise this as a limitation of our research (21).

In our population, anti-SSA antibodies demonstrated higher values in patients with diffuse cutaneous involvement. However, we did not find significant associations with organ involvement in scleroderma patients, similar to other studies (22).

Male patients appeared more prone to the development of DUs in our SSc cohort. These findings mirror those found in recent litterature, male gender being a known risk factor for SSc-related digital trophic lesions $(23,24)$.

Senescence is a biological process affecting all organs and systems. In light of recent discoveries, telomere alterations consequent to repeated cell divisions are responsible for the age-related decline of cell function. In this regard, the immune system also suffers from age-related changes, elderly patients demonstrating a reduced capability of antibody production resulting in a reduced response to vaccination and an increased risk of infection (25).

The impact of immunosenescence on the dynamics of autoantibody production in CTD remains in need of further investigation. Although elderly patients with SSc did not demonstrate a significantly decreased autoantibody production, lupus patients over 60 years of age exhibited lower anti-nucleosome antibody titers in the present study (26).

Moreover, elderly scleroderma patients were more likely to exhibit PAH and ILD in our cohort. 
These findings resemble those described by other research teams $(27,28)$.

\section{CONCLUSIONS}

Connective tissue diseases constitute a complex group of conditions in which immune disturbance is invariably present. Recent research provides new insights on the pathogenic processes of autoimmune

\section{REFERENCES}

1. Soto ME, Hernández-Becerril N, Perez-Chiney AC et al. Predictive value of antinuclear antibodies in autoimmune diseases classified by clinical criteria: Analytical study in a specialized health institute, one year follow-up. Results Immunol. 2015;5(1):13-22.

2. Barturen $G$, Beretta $L$, Cervera $R$ et al. Moving towards a molecular taxonomy of autoimmune rheumatic diseases. Nature Rev Rheumatol. 2018;14(2):75-93.

3. Gigante $\mathrm{A}$, Rosato $\mathrm{E}$, Liberatori $\mathrm{M}$ et al. Autonomic dysfunction in patients with systemic sclerosis: correlation with intrarenal arterial stiffness. Int J Cardiol. 2014;177(2):578-80.

4. Hoogen F, Khanna D, Fransen J et al. 2013 classification criteria for systemic sclerosis: an American College of Rheumatology/ European League against Rheumatism collaborative initiative. Arthritis Rheumatol. 2013;65(11):2737-47.

5. Denton CP, Krieg T, Guillevin L et al. Demographic, clinical and antibody characteristics of patients with digital ulcers in systemic sclerosis: data from the DUO Registry. Ann Rheum Dis. 2012;71(5):718-21..

6. Sato $\mathrm{S}$, Kodera M, Hasegawa $\mathrm{M}$ et al. Antinucleosome antibody is a major autoantibody in localized scleroderma. $\mathrm{Br} \mathrm{J}$ Dermatol. 2004;151(6):1182-8.

7. Agarwal S, Busse PJ. Innate and adaptive immunosenescence. Ann Allergy Asthma Immunol. 2010;104(3):183-90.

8. Burlui A, Graur M, Gherasim A et al. The role of adipokines in connective tissue diseases: can we face the challenge? Int J Med Dent, 2018 22(2):132-9.

9. Mahler M, Meroni PL, Bossuyt X, Fritzler MJ. Current concepts and future directions for the assessment of autoantibodies to cellular antigens referred to as anti-nuclear antibodies. J Immunol Res. 2014;21(1):1-19.

10. Olsen NJ, Choi MY, Fritzler MJ. Emerging technologies in autoantibody testing for rheumatic diseases. Arthritis Res Ther. 2017;19(1):172-82.

11. Joseph CG, Darrah E, Shah AA et al. Association of the autoimmune disease scleroderma with an immunologic response to cancer. Science. 2014;343(6167):152-7.

12. Silva I, Almeida J, Vasconcelos C. A PRISMA-driven systematic review for predictive risk factors of digital ulcers in systemic sclerosis patients. Autoimmun Rev. 2015;14(2):140-52.

13. Liaskos C, Marou E, Simopoulou T et al. Disease-related autoantibody profile in patients with systemic sclerosis. Autoimmunity. 2017;50(7):414-21.

14. Choi MY, Fritzler MJ. Progress in understanding the diagnostic and pathogenic role of autoantibodies associated with systemic sclerosis. Curr Opin Rheumatol. 2016;28(6):586-94. rheumatic diseases, in an attempt to identify potential risk factors for organ involvement. Our study identified a possible link between anti-nucleosome antibodies and cardiopulmonary involvement in the SSc population, similar to other studies. Nevertheless, this relationship is currently gathering interest from the scientific community.

\section{Conflict of interest: none declared Financial support: none declared}

15. Lian F, Chen D, Wang $Y$ et al. Clinical features and independent predictors of pulmonary arterial hypertension in systemic lupus erythematosus. Rheumatol. Int. 2012;32(6):1727-31.

16. Sui $M, L i n Q, X u Z$ et al. Simultaneous positivity for anti-DNA, anti-nucleosome and anti-histone antibodies is a marker for more severe lupus nephritis. J Clin Immunol. 2013;33(2):378-87.

17. Mjelle JE, Rekvig OP, Van Der Vlag J, Fenton KA. Nephritogenic antibodies bind in glomeruli through interaction with exposed chromatin fragments and not with renal cross-reactive antigens. Autoimmunity. 2011;44(5):373-83.

18. Hesselstrand R, Scheja A, Shen GQ et al. The association of antinuclear antibodies with organ involvement and survival in systemic sclerosis. Rheumatology 2003;42(4):534-40.

19. Mehra S, Walker J, Patterson K, Fritzler MJ. Autoantibodies in systemic sclerosis. Autoimmun Rev. 2013;12(3):340-54.

20. Komócsi A, Vorobcsuk A, Faludi $R$ et al. The impact of cardiopulmonary manifestations on the mortality of SSc: a systematic review and meta-analysis of observational studies. Rheumatology. 2012;51(6):1027-36.

21. Sanchez-Montalva A, Fernandez-Luque A, Simeon CP et al. Anti-SSA/Ro52 autoantibodies in scleroderma: results of an observational, cross-sectional study. Clin Exp Rheumatol. 2014;32(6 Suppl 86):S-177.

22. Manfredi A, Sebastiani M, Carraro V et al. Prediction risk chart for scleroderma digital ulcers: a composite predictive model based on capillaroscopic, demographic and clinico-serological parameters. Clin Hemorheol Microcirc. 2015 Jan 1;59(2):133-43.

23. Doherty M, Schmidt-Ott R, Santos Jl et al. Vaccination of special populations: Protecting the vulnerable. Vaccine. 2016; 34(52):6681-90

24. Groseanu L, Cambur I, Balanescu A, lonescu R. Digital ulcers and multiple amputations in a systemic sclerosis patient. Romanian $\mathrm{J}$ Rheumatol. 2017;26(4):173-8.

25. Nihtyanova SI, Schreiber BE, Ong VH et al. Prediction of pulmonary complications and long term survival in systemic sclerosis. Arthritis Rheumatol. 2014;66(6):1625-35.

26. Liang KP, Gabriel SE. Autoantibodies: Innocent bystander or key player in immunosenescence and atherosclerosis? J Rheumatol. 2007;34(6):1203-7.

27. Muresan L, Petcu A, Muresan C et al. Evaluation of cardiac autonomic function with holter ecg monitoring in patients with systemic sclerosis. Romanian J Rheumatol. 2015;24(3):152-9.

28. Kayser C, Fritzler MJ. Autoantibodies in systemic sclerosis: unanswered questions. Front Immunol. 2015;6:167. 